\title{
Reflections on Bilateral Cochlear Implants
}

\author{
Marcia Yuri Tsumura Kimura', Miguel Angelo Hyppolito ${ }^{2}$ \\ ${ }^{1}$ Postgraduate Program in Oftalmology, Otorrhinolaryngology and Head Neck Surgery of the FMRP/USP-Medical Sciences, De- \\ partment of ophthalmology, Otorhinolaryngology and Head and Neck Surgery, Faculty of Medicine of Ribeirão Preto, University of \\ São Paulo, São Paulo, Brazil; ${ }^{2}$ Departament of Ophthalmology, Otorhinolaryngology and Head and Neck Surgery, Faculty of Medi- \\ cine of Ribeirão Preto, University of São Paulo, São Paulo, Brazil. \\ Email: marcia.kimura@politecsaude.com.br, mahyppo@fmrp.usp.br
}

Received December $10^{\text {th }}, 2012$; revised January $26^{\text {th }}, 2013$; accepted March $14^{\text {th }}, 2013$

\begin{abstract}
The bilateral cochlear implant has increased in recent years in due to the search for auditory enhancements. There are many advantages to the users of the bilateral cochlear implant, which may include the location of the auditory sign, decreasing the head shadow effect for the contralateral ear and binaural summation of the hearing. In children it is also discussed the issue of auditory deprivation. The aim of this review was to present a reflection on issues related to the bilateral cochlear implant, allowing the reader to do a search and strengthen scientifically with this issue, giving theoretical foundation to better guide and advise their patients.
\end{abstract}

Keywords: Bilateral Cochlear Implants; Hearing Loss; Auditory Performance

\section{Introduction}

Hearing is one of the most important senses for human life. It is the key to the oral language acquisition and a way to feel the world around them. We realize that without it, the individual loses part of the real world, experiencing emotional and social problems.

Any subject that is not exposed to stimulation of language in the first years of life will present a lag in their auditory and linguistic development. The first years of life are considered critical for the development of auditory and language skills. It is the period of greatest neuronal plasticity in the auditory pathway. During this period, the central auditory nervous system can be changed positively or negatively, depending on the quantity and quality of external stimuli picked up. In addition, the period of receipt of hearing linguistic symbols is a prerequisite to form the oral communication [1].

The audiological diagnosis carried out during the first year of life provides early medical and/or audiological intervention, still within this critical period, allowing a more favorable prognosis in relation to the overall development of the child. That is, the sooner the intervenetion occurs, the shorter the time of deprivation and consequently better the results with early rehabilitation.

It is well established that the changes resulting from hearing loss restrict the entry of sounds that will change the auditory development and consequently the language.

In most cases of sensory-neural hearing loss, the first site of lesion is inside the cochlea, where the membranous labyrinth is. Cochlear hearing loss results in insuffi- cient energy transduction of the acoustic mechanism of neural impulses to the auditory nerve. Currently, we have available the electronic device "cochlear implant".

The cochlear implant is considered the only high-tech device capable of converting acoustic signals into electrical stimuli causing auditory sensation through direct stimulation of the auditory nerve. It is considered the most effective sensory prosthesis in the history of medicine. It is characterized by having an internal part (consisting of a bundle of electrodes, receiver-stimulator and internal magnet), which is surgically inserted and an outside part (composed of a speech processor, microphone and external antenna) that the user carries with them (Figure 1).

The speech processor stores the "heart" of the cochlear implant that is the speech coding strategy, responsible for converting all acoustic information captured by the microphone into electrical signals that are sent to the internal drive and thus stimulate the auditory nerve [2].

The technology of the cochlear implant is indicated for people with severe-deep sensory-neural or deep bilateral deafness not presenting benefits with conventional hearing aids, Table 1. Evaluation for cochlear implant system must be carried out by an interdisciplinary team, which evaluates medical, phonoaudiological, social, and psychological of the candidate [2].

Cochlear implant surgery is performed under general anesthesia through a small retroauricular incision $(6 \mathrm{~cm})$ with a tympanomastoidectomy being required in most cases a posterior tympanotomy and a cochleostomy in 
the round window or in promontory with the objective to insert the electrodes in inner ear tympanic canal.

The Cochlear implant complications are rare and may be divided into early and late complications or medical and surgery complications and complications related to the equipment. The late complications occur over 3 months of surgery. Early complications are common and the same for children and adults, with rates of $0.2 \%$ to $2.5 \%$. The most frequent complications are related to the myocutaneous flap, displacement of the electrodes, problems with the facial nerve and improper insertion of electrodes, especially in cases of malformations and cochlear ossification intra-cochlear post meningitis.

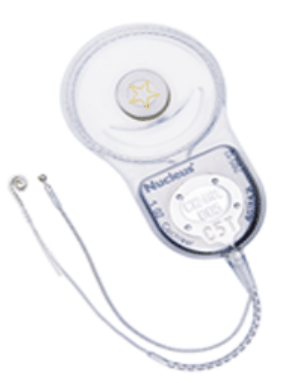

(a)

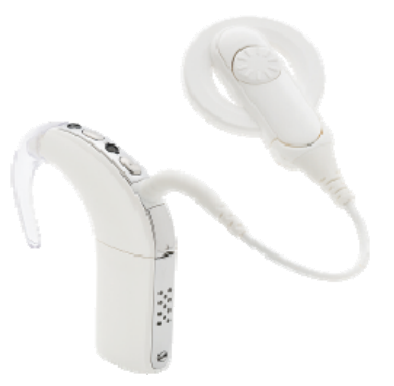

(b)
Figure 1. Cochlear implant system: (a) Internal component (Nucleus CI24RE (CA)) and (b) External component (Nucleus $\mathbf{C P 8 1 0 )}$. Photos courtesy of Cochlear $^{\mathrm{TM}}$.

Table 1. Indications to unilateral or bilateral cochlear implant in adults and children and special situations.

\section{Bilateral Cochlear Implant}

\section{Adults and Children}

- Bilateral cochlear implant simultaneously for all children with severe to profound bilateral hearing loss as soon as possible when the definitive diagnosis of deafness.

- In cases of sequential cochlear implant, the second implant must be carried out in an interval of less than 1 year.

- Researches have shown that children would have greater benefit up to 4 years old with bilateral cochlear implant, however $50 \%$ of children between 4 and 7 years would benefit with the bilateral cochlear implant.

- Simultaneous or sequential bilateral cochlear implant must be indicated by the 7 years old when there is bilateral severe or severe to profound sensorineural hearing loss; auditory threshold above $80 \mathrm{~dB}$ in 1,2,3 and $4 \mathrm{kHz}$ in the better ear without benefit with conventional hearing aid; absence of cognitive impairment and autism.

- Individuals older than 7 years with prelingually deafness, sequential bilateral cochlear implant should be considered and indicated when occur bilateral severe to profound sensorineural hearing loss; auditory threshold above $80 \mathrm{~dB}$ in 1,2,3 and $4 \mathrm{kHz}$ in the better ear; presence of linguistic code established; first implant performed up to 2 years of age and interval between the first and second implant not exceeding 7 years; absence of cognitive impairment and autism.

- Individuals with Postlingually hearing loss should be considered in bilateral severe to profound sensorineural hearing loss; auditory threshold above $80 \mathrm{~dB}$ in 1,2, 3 and $4 \mathrm{kHz}$ in the better ear; use of cochlear implants in the first ear for at least 1 year (except in cases of meningitis with less than 2 years of deafness).

- Individuals with hearing loss and blindness, the simultaneous or sequential bilateral cochlear implant should be considered when the bilateral severe or severe to profound sensorineural hearing loss; auditory threshold above $80 \mathrm{~dB}$ in 1,2,3 and $4 \mathrm{kHz}$ in the better ear.

Unilateral Cochlear Implant

Adults Children

- Postlingually deaf adults with speech discrimination score lower than $40 \%$.

- Open-set CVC-words scored on words at $65 \mathrm{~dB} \mathrm{HL}$ in quiet.

- Profound or severe to profound hearing loss without marginal gain from acoustic amplification.

- Residual hearing for bass sound frequencies.

- Prelingually deaf adults with some "peripheral cochlear hearing" that provide identification of environmental sounds and may support speech reading.

- Language development, previous experience with hearing.

- Motivation to CI use.

- Profound or severe to profound bilaterally hearing loss with average pure tone threshold in higher than $85 \mathrm{~dB}$ without hearing aid benefit when language development in clearly retarded (on this condition children should be implanted as early as possible to decrease the duration of auditor deprivation).

- Prelingually children with profound or severe to profound bilaterally hearing loss with average pure tone threshold in higher than $85 \mathrm{~dB}$ that occoured less than the age of 2 years.

- Absence of cognitive impairment and autism.

\section{Uncommon Indications}

- Malformed cochlea is variable and depends on the type of malformation, with auditory nerve present.

- Acquired dysmorphia of inner ear or cochlear nerve. Some cases of cochlear ossification due to pneumococcal meningitis.

- Auditory neuropathy.

- Associated disabilities (Blindness, motor disturbances, mental retardation, epilepsy, skeletal malformations). Associated disabilities do not exclude $\mathrm{CI}$ but depends on the brain damage that can impede hearing outcome.

\section{Other Emerging Indications}

- Residual hearing in contralateral ear, non implanted ear, may be aided by a conventional hearing aid, providing the adult or child with binaural hearing. 
Medical and surgery complications are related to the scalp in the region of the bed of deployment of internal unity of the implant, infectious such as acute otitis media and meningitis, changes the function of the facial nerve, disorders of the vestibular system and migration of implanted system. Complications related to equipment deployed are related to the failure of the same and the stimulation of the facial nerve.

The results of people who use cochlear implants show that it allows listening sensation and access to oral language, allowing their users acquisition and development of auditory and language skills. From these results it is important to note that, to be introduced in the human being, the variability in the results is evident. An important detail and worthy of emphasis is the fact that the earlier the intervention occurs, the better the result reached with the use of the cochlear implant [3].

The success of the cochlear implant depends on the speech processor programming. The programs will determine how the cochlear implant will send to the auditory nerve electrical stimulation of the sounds from the speech and environmental sounds picked up by the microphone. It is important to determine the level of required electrical stimulation to restore weak hearing sounds and achieve an equalized adjustment independent of the level of intensity of sound input [2].

The first cochlear implant approved by FDA (Food and Drug Administration) occurred in 1985 (Zwolan, 2000), initially for deployment of this prosthesis in adults with post-lingual deafness (hearing loss gained after the acquisition of language) and only after ten years was approved the cochlear implant in adults with pre-lingual deafness (hearing deficiency present at birth or acquired by any etiology established after birth and before 4 years old (Kessler and Owens, 1989).

Developments in the indication criteria suffer direct interference of the constant technological development along with the improvement of surgical techniques and training and qualification of interdisciplinary teams.

From the first approval until today, one of the most obvious changes was related to the age to receive the cochlear implant that being approved for pre-lingual children, their age was 24 months. Currently, the indication is from 6 months, thereby decreasing the time of deprivation and consequently better auditory and oral language performance $[3,4]$.

For Geers (2004) [5] and Nicholas, Geers (2007) [6], children who receive the cochlear implant early often present an appropriate oral language development which is similar to the child who does not present any deficiency in the hearing.

The current discussion is regarding the indication of bilateral cochlear implant. Studies have shown that the use of unilateral cochlear implant provides hearing, however the user presents difficulties as to the location and discrimination of sounds in noisy environments, and requires a greater effort to track the conversation [7]. In addition, there are doubts as to the time of surgery for bilateral sequential or simultaneous implantation, Table 1.

\section{Bilateral Cochlear Implant}

It is a characteristic of the current way of life to coexistence among individuals in noisy environments. Humans spend most of their time in environments containing various sounds and the auditory system is faced with the important task of identifying and locating sound sources. It is believed that in listeners with normal hearing, the binaural system is very important to provide clues that allow this to occur with fidelity [8].

Two issues should be considered when we are evaluating the child for bilateral cochlear implant regardless of their age. The age of the child itself could affect the auditory development independently, but there is no evidence that proves so.

Binaural stimulation results in a process in which the input stimuli in both ears are integrated into the auditory pathways and encoded, allowing the external perception and favors its spatial location. In addition, the sounds are segregated in separate images that can carry information about location and content. With the entry of the stimulus in one ear, the sound location becomes difficult $[8,9]$.

The benefits of binaural stimulation were observed in the study of Hawley et al. (2004) [10] as for the improvement in the ability to understand speech in the presence of competitive sounds, because they are able to take advantage of the interaural and spatial cues.

The role of binaural hearing in the clinic population has been a topic of great interest, in particular in the cochlear implant users. Although the cochlear implant has obtained clear success in providing hearing entry in deaf people, limitations on the information that it is able to provide are evident. When an individual uses a unilateral cochlear implant, one of the limitations is the ability to perceive multiple entries with segregated independent sources. This reflects the difficulty in understanding speech in the presence of competitive signals and identify the location of the sound in the environment.

Aiming at an improvement in speech understanding difficulties in the presence of noise and localization of sound source, thousands of patients have sought the bilateral cochlear implant [11].

Regarding the location of sound, a number of studies have demonstrated better performance resulting from use of the bilateral cochlear implant and how to identify sounds within a multi-speaker matrix, usually resulting in fewer errors when patients with bilateral cochlear implant are evaluated. However, one variation within groups 
is observed $[10,12,13]$.

The magnitude and type of advantage of the cochlear implant are not universal. Three primary advantages can be measured when comparing the performance of unilateral versus bilateral hearing conditions, which are: head shadow effect, squelch effect and the binaural summation. These effects contribute significantly to improve speech understanding, especially in environments with noise and on the location of the sound, because it allows the central auditory system to calculate difference of minutes in the characteristics of sound arrival in each ear.

The head shadow effect is a physical phenomenon which occurs when the head blocks the arrival of sound in the ear from different locations. This effect allows the listener to hear using the ear with better signal-to-noise ratio (SNR). The binaural redundancy or binaural summation is the result of central auditory processing that occurs when entries in both ears are analyzed throughout the hearing way. This phenomenon demonstrates the ability of the auditory nervous system to integrate and use the information from two ears, allowing better performance than a single ear. The squelch effect is the ability of the auditory system to use the information of both ears when speech and noise are spatially separated and uses the advantage of aggregating the ear with worse signal-to-noise ratio [14].

Litovisky et al. (2004) [15] showed that there is a correlation between the tasks of intelligibility and sound location, i.e. bilateral cochlear implant users that are able to locate sounds are also capable of understanding speech in noisy situations. The possibility is that, for those individuals in which both the performance of speech intelligibility and location were good, it shows that the aspects of binaural hearing were being used in such a way as to facilitate their performance. However, it is not possible to determine what are the exact binaural mechanisms involved in each task. We only know that in the task of speech intelligibility, there is the "better ear effect", where the stimulus is increased to the best ear due to noise abatement by the head.

In general, the indications for the bilateral cochlear implant are:

Children above 5 and 18 months of age with bilateral sensorioneural hearing loss severe to profound, auditory threshold above $80 \mathrm{~dB}$ in the better ear, absence of cognitive impairment and autism.

In individuals older than 5 years consider bilateral sensorineural hearing loss severe to profound, auditory threshold above $80 \mathrm{~dB}$ in the better ear, presence of linguistic code established, first implant performed up to 2 years of age and interval between the first and second implant not exceeding 5 years and no cognitive deficit and autism.

In individuals with post-lingual hearing loss should be consider the sensorineural hearing loss severe to profound bilateral; auditory threshold above $80 \mathrm{~dB}$ in the better ear; use of cochlear implants in the first ear for at least 1 year (except in cases of meningitis with less than 2 years of deafness).

In individuals with hearing loss and blindness should be consider the sensorioneural hearing loss severe to profound bilateral with auditory threshold above $80 \mathrm{~dB}$ in the better ear.

\subsection{Simultaneous or Sequential Bilateral Cochlear Implant?}

There is a critical period for the binaural auditory development, more favorable for early simultaneous deployment. Studies of Bauer et al. (2006) [16] and Gordon et al. (2007) [17] showed evidence of plasticity of central auditory pathways soon after early bilateral implantation and clear differences were observed in the electrophysiological studies in children implanted bilaterally in sequence even when the second implant was performed in a time interval shorter than 1 year.

The study of Wie (2010) [4] showed that users who have been subjected to simultaneous bilateral surgery between 5 and 18 months of age and after 9 to 12 months of use of cochlear implant achieved a result of auditory and language development equal to the listener children with the same chronological age. It has been concluded that children with pre-lingual deafness may develop oral expressive and receptive language within the normal range if implanted early. This is possible because the function of the cerebral cortex will have a very small and minimum delay and in such cases [18].

In adults, studies have shown that the simultaneous or sequential bilateral cochlear implant improves comfort of listening with two ears and provides additional benefit to the arrival of auditory information, besides the location of sound, proven by the fact that the two ears are getting auditory sensations [19].

Initial data suggest that binaural measurable benefit (by psychophysical and electrophysiological measurement) of the sequential cochlear implant in children will decrease in function of the interval between implants. Concurrency and the small gap between the realization of cochlear implants should be considered for the best rehabilitation and greater opportunity for the development of speech and language, minimizing the range of bilateral deafness [20].

In the study of Gantz et al. (2010) [21], substantial benefits in word recognition and location were found within the first 12 months after cochlear implants and continued to occur in ascending scale with improvement in hearing over time.

One important aspect to be highlighted is that the simultaneous bilateral surgery reduces the intervention 
time for the two surgeries than one in each moment, a single general anesthesia and reduction in clinical care. It is important that the medical staff is prepared to reduce surgical time as much as possible. In children appropriately selected there are no additional surgical risks in simultaneous surgeries. In sequential surgeries the risks are the same, but the patient will be subjected to risk twice [22].

\subsection{Age for Bilateral Cochlear Implant}

The bilateral cochlear implant could be considered standard for children with deep-severe sensory-neural and deep bilateral hearing impairment [20].

Two issues are important when considering bilateral cochlear implant in children: the time of deafness prior to the first surgery, which interferes with the development of oral language and linguistic skills; and the interval between the surgeries, which possibly affects the development of binaural processing [20].

The time of indication and the age to be subjected to surgery should be seriously discussed with the cochlear implant program team, which will have as its goal an early intervention in search of better hearing conditions in every way, providing hearing benefits in search of respect and social inclusion.

Papsin and Gordon (2008) [20] emphasized that if the financial issue is not a problem, the bilateral cochlear implant would benefit all children with deep bilateral sensory-neural deafness, with the caveat that it will not be subject to additional risks and both ears should meet the pediatric audiometric criteria. It has also been reported that if the benefit of the second implant is small, it is not worth the cost, and that health systems and doctors will consider the cost-effectiveness issue.

The greatest contraindication of bilateral cochlear implant is the body weight of the child under $6 \mathrm{~kg}$ and the lack of family support. For adults, the contraindications are related to time of sound deprivation (deafness) without use of individual sound amplification equipment.

\subsection{Reflections on New Technologies}

We cannot forget that there is a critical period for the cerebral cortical development, so the early intervention of cochlear implant should occur as soon as possible and consequently the second surgery as well. Given this, waiting for a new technology for a future hearing intervention may not contribute to the development, once the cortex may not take advantage of the benefits of this new technology.

\subsection{Which Device Brand Should One Choose on the Second Cochlear Implant?}

Some studies have shown that different technologies and different speech coding strategies in each ear does not affect performance in the results. Budenz et al. (2009) stated that the sequential bilateral cochlear implant with new or different technology does not diminish the benefit of bilateral stimulation. They still reported that it may improve after the second implant, regardless of the device selected, and after a period of adjustments, they have good development, even with different processors and strategies.

The issue of different models of speech processors, in cases of different brands and technologies, one must be oriented to check if there is really no aesthetic factor that can interfere in this selection.

There is still a factor of convenience in relate to only one brand of cochlear implant, rather than two, in times of search for repairs, buying accessories, maintenance.

The possible options should always be discussed with the interdisciplinary team before any doubt or decision. It is important that all options are explained clearly to the patient and his family, explaining all procedures related to cochlear implant.

\subsection{Hearing Rehabilitation on Bilateral Cochlear Implant Users}

The following text is based on the work "Therapy and educational guidelines for bilateral cochlear implantation", a material prepared and provided by Cochlear Corporation (2007).

The outcomes with the bilateral implantation are: the understanding of speech and functional integration of each implant resulting in a balanced binaural hearing. This requires an effective hearing rehabilitation work, but not very different from the process of rehabilitation of a single cochlear implant.

Some fundamental concepts are important to remember, such as:

- Each user is unique and there are factors that affect the end result.

- Create positive auditory experiences and select appropriate activities for every hearing age and skill.

- Adults should always carry extra batteries. Parents and teachers should always have extra batteries available.

- Introduce the hearing aid devices.

- Demonstrate the importance of hearing practice, through interactions of structured natural speech and hearing therapy.

Below are some suggestions for therapy and educational process:

- Check separately each ear using the Ling Sounds or auditory discrimination activities until the user is able to identify when one of the implants isn't working or when the batteries are dead. 
- Establish specific goals for each ear separately or for binaural hearing based on speech acoustics and auditory development. Check if parents or users understood the goals and reasons. Use these objectives to monitor progress and inform the user of the improvements in auditory skills.

- Attention to the practice in the step where the user is in recommended individual therapy.

- Expect the following:

- Recognition in closed to open set.

- Recognition from predictable to unpredictable information.

- Recognition of familiar to unfamiliar words.

- Use of repetition to non-repetition.

- Recognition of close to more distant sounds.

- Recognition in quiet to noisy environments.

Unique considerations on the rehabilitation in cases of Bilateral Cochlear Implant:

1) Simultaneous Cochlear Implant

With the simultaneous implantation, the user uses two implants all the time. If, however, there is a great discrepancy between the two ears, is advised to work development of he worse ear at a specific time. Usually this separate work is not necessary.

2) Sequential Cochlear Implant

After sequential implementation, listening with "the new ear" is needed to get the maximum benefit from the bilateral cochlear implant to balance the second hearing competence with the first implant. The goal of rehabilitation in sequential implementation is not only the second implant recovery so that it is equivalent to the first, but the integration of the two ears, so that together they contribute to the daily hearing [23].

Sometimes, the second implant can be better than the first. Initially, encourage them to use only the new implant for a few hours of the day in quiet environments and hearing therapy is made with the new implant all or part of it, depending on the stage of rehabilitation and the user's age.

Continue in this manner until the score of speech perception of the second implant is next to the first, or until the user is in speech recognition in open set in quiet environment. For some users, the second implant will never reach the first and after some time, hearing with both ears must prevail to the isolated performance.

Therapy and suggestions on sequential implementation:

- Advise parents and users that the initial hearing perception of the implanted ear must be basic level and not advanced skills as the first implant.

- General clinical trials indicate that progress is faster with the second than the first implant.

- Make sure both implants are being used every day, in all environments and especially to hear guidance, news, or complex information.

- Create situations to demonstrate the benefit of two implants as a simple task of location, noise or speech test with different hearing situation environments.

- If the time between the first and the second implant is of a few months, use both all the time.

- If the time between the first and the second implant is long, use only the new implant for a few hours as well as in therapy.

- If the child is under 3 years of age, it is recommended to use both implants all the time. If it is observed that there is no perception or auditory discrimination, it is recommended a hearing practice training in a short and fast period with the newer implant or with the worst result.

\section{Conclusions}

Early bilateral cochlear implantation results in the preservation of the central auditory system.

The indication of the bilateral cochlear implant must be discussed with the interdisciplinary team and always considered when there are no significant risks.

The arrival of auditory information on both ears is extremely important for understanding speech, especially in situations of great challenge, like music.

Several studies have shown the total benefit of "head shadow effect" and binaural summation effect in bilateral cochlear implant users.

There is better speech recognition in noise and better localization with bilateral cochlear implant compared to a single implant.

\section{Acknowledgements}

We wish to thanks Cochlear $^{\mathrm{TM}}$ for Photos.

\section{REFERENCES}

[1] A. L. M. Moret, M. C. Bevilacqua and O. Costa Filho, "Implante Coclear: Audição e Linguagem em Crianças Deficientes Auditivas Pré-Linguais," Pro-Fono Revista de Atualização Científica, Vol. 19, No. 3, 2007, pp. 295304. doi:10.1590/S0104-56872007000300008

[2] M. C. Bevilacqua, O. Costa Filho and A. C. Martinho, "Implante Coclear," In: Tratado de Fonoaudiologia, Roca, 2004, pp. 751-761.

[3] D. M. Valencia, F. L. Rimell, B. J. Friedman, M. R. Oblander and J. Helmbrecht, "Cochlear Implantation in Infants Less than 12 Months Ages," International Journal of Pediatric Otorhinolaryngology, Vol. 72, No. 6, 2008, pp. 767-773. doi:10.1016/j.ijporl.2008.02.009

[4] O. B. Wie, "Language Development in Children after Receiving Bilateral Cochlear Implants between 5 and 18 Months," International Journal of Otolaryngology, Vol. 74, No. 11, 2010, pp. 1258-1266.

[5] A. E. Geers, "Speech, Language and Reading Skills after 
Early Cochlear Implantation," Archives of Otolaryngology-Head and Neck Surgery, Vol. 130, No. 5, 2004, pp. 634-663. doi:10.1001/archotol.130.5.634

[6] J. G. Nicholas and A. E. Geers, "Will They Catch Up? The Role of Age at Cochlear Implantation in the Spoken Language Development of Children with Severe to Profund Hearing Loss," Journal of Speech, Language, and Hearing Research, Vol. 50, No. 4, 2007, pp. 1048-1062. doi:10.1044/1092-4388(2007/073)

[7] R. Y. Litovsky and S. P. Godar, "Experience with Bilateral Cochlear Implants Improves Sound Localization Acuity in Children," Otology \& Neurotology, Vol. 31, No. 8, 2010, pp. 1287-1292.

[8] J. Blauert, "Spatial Hearing Cambridge," The MIT Press, Massachusetts, 1997.

[9] M. L. Hawley, R. Y. Litovsky and H. S. Colburn, "Speech Intelligibility and Localization in Complex Environments," Journal of the Acoustical Society of America, Vol. 105, No. 6, 1999, pp. 3436-3448. doi: $10.1121 / 1.424670$

[10] M. L. Hawley, R. Y. Litovsky and J. F. Culling, "The Benefit of Binaural Hearing in a Cocktail Party: Effect of Location and Type of Interferer," Journal of the Acoustical Society of America, Vol. 115, No. 2, 2004, pp. 833843. doi:10.1121/1.1639908

[11] R. S. Tyler, C. C. Dunn, S. A. Witt and J. P. Preece, "Update on Bilateral Cochlear Implantation," Current Opinion in Otolaryngology \& Head and Neck Surgery, Vol. 11, No. 5, 2003, pp. 388-393.

[12] D. W. Grantham, D. H. Ashmead, T. A. Ricketts, et al., "Horizontal-Plane Localization of Noise and Speech Signals by Postlingually Deafened Adults Fitted with Bilateral Cochlear Implants," Ear and Hearing, Vol. 28, No. 4, 2007, pp. 524-541. doi:10.1097/AUD.0b013e31806dc21a

[13] A. C. Neuman, A. Haravon, N. Sislian, et al., "Sound Direction Identification with Bilateral Cochlear Implants," Ear and Hearing, Vol. 28, No. 1, 2007, pp. 73-82. doi:10.1097/01.aud.0000249910.80803.b9

[14] E. C. Schafer, A. M. Amlani, A. Seibold and P. L. Shattuck, "A Meta-Analytic Comparison of Binaural Benefits between Bilateral Cochlear Implants and Bimodal Stimulation," Journal of the American Academy of Audiology,
Vol. 18, No. 9, 2007, pp. 760-776.

[15] R. Y. Litovsky, A. Parkinson, J. Arcaroli, et al., "Bilateral Cochlear Implants in Adults and Children," Archives of Otolaryngology—Head \& Neck Surgery, Vol. 130, No. 4, 2004, pp. 648-655. doi:10.1001/archotol.130.5.648

[16] P. W. Bauer, A. Sharma and M. Dorman, "Central Auditory Development in Children with Bilateral Cochlear Implants," Archives of Otolaryngology-Head \& Neck Surgery, Vol. 1132, No. 10, 2006, pp. 1133-1136.

[17] K. A. Gordon, J. Valero and B. C. Papsin, "Binaural Processing in Children Using Bilateral Cochlear Implants," NeuroReport, Vol. 18, No. 6, 2007, pp. 613-617. doi:10.1097/WNR.0b013e3280b10c15

[18] B. C Papsin, J. D. Ramsden, R. Leung, A. James and K. A Gordon, "Bilateral Simultaneous Cochlear Implantation in Children: Our First 50 Cases," Laryngoscope, Vol. 119, No. 12, 2009, pp. 2444-2448.

[19] M. Mok, K. C. Hughes and K. L. Galvin, "Can Adolescents and Young Adults with Prelingual Hearing Loss Benefit from a Second, Seqüencial Cochlear Implant," International Journal of Audiology, Vol. 49, No. 5, 2010, pp. 368-377.

[20] B. C. Papsin and K. A. Gordon, "Bilateral Cochlear Implants Should Be the Standard for Children with Bilateral Sensorineural Deafness," Otolaryngology \& Head and Neck Surgery, Vol. 16, No. 1, 2008, pp. 69-74.

[21] B. J. Gantz, C. C. Dunn, W. Noble, R. S. Tyler, M. Kordus and $\mathrm{H}$. Ji, "Bilateral and Unilateral Cochlear Implant Users Compared on Speech Perception in Noise," Ear and Hear, Vol. 31, No. 2, 2010, pp. 296-298. doi:10.1097/AUD.0b013e3181c12383

[22] J. Kronenberg and L. Migirov, "Bilateral, Simultaneous Cochlear Implatation in Children: Surgical Considerations," Journal of Laryngology \& Otology, Vol. 123, No. 8, 2009, pp. 837-839.

[23] H. Kuhn-Inacker, W. Shehata-Dieler, J. Muller and J. Helms, "Bilateral Cochlear Implants: A Way to Optimize Auditory Perception Abilities in Deaf Children?" International Journal of Pediatric Otorhinolaryngology, Vol. 68, No. 10, 2004, pp. 1257-1266. doi:10.1016/j.ijporl.2004.04.029 